\title{
Asymmetry of the Aharonov-Bohm diffraction pattern and Ehrenfest's theorem
}

\author{
Donald H. Kobe \\ Department of Physics, University of North Texas, Denton, Texas 76203-5368 \\ Valdir C. Aguilera-Navarro and Regina M. Ricotta \\ Instituto de Física Teórica, UNESP, Rua Pamplona 145, 01405 São Paulo, São Paulo, Brazil
}

(Received 23 September 1991)

\begin{abstract}
The electron-diffraction pattern for two slits with magnetic flux confined to an inaccessible region between them is calculated. The Aharonov-Bohm effect gives a diffraction pattern that is asymmetric but has a symmetric envelope. In general, both the expected displacement and the kinetic momentum of the electron are nonzero as a consequence of the asymmetry. Nevertheless, Ehrenfest's theorems and the conservation of momentum are satisfied.
\end{abstract}

PACS number(s): 03.65.Bz, 03.65.Nk

\section{INTRODUCTION}

Over 30 years ago Aharonov and Bohm (AB) [1] showed that in quantum mechanics the vector potential plays a role very different from classical physics. In classical electrodynamics the vector potential is only a mathematical tool to solve Maxwell's equations. However, quantum mechanics is a Hamiltonian theory which involves the vector potential in order to have a local interaction between the electron and the field. The Schrödinger equation is, nevertheless, gauge covariant. A gauge transformation on the vector potential and multiplication of the wave function by a corresponding phase factor leaves the Schrödinger equation form invariant. However, when a nontrivial topology is involved, the phase factor plays an essential role. Wu and Yang [2] put the situation succinctly when they said that in quantum mechanics the electromagnetic field is an underdescription of electromagnetic phenomena, while the use of the vector potential is an overdescription.

For a two-slit diffraction experiment with magnetic flux confined to an inaccessible region between the two slits, the vector potential gives a relative phase difference between the amplitudes for the electron to pass through each slit. This relative phase, which depends on the flux enclosed, causes a shift in the two-slit diffraction pattern within the unshifted envelope. The diffraction pattern is asymmetric in general, except for certain values of the flux. If more electrons were scattered to the right, for example, than to the left, how can this asymmetry be explained? Since the magnetic field of the solenoid between the slits is completely shielded, there is no Lorentz force acting on the electron. Therefore, there appears to be a violation of Ehrenfest's theorem. The commonly accepted point of view is that even though the diffraction pattern is asymmetric, the average displacement of the electrons and consequently their average kinetic momentum is zero [3-5].

A previous calculation [6] of the diffraction pattern using slits with a Gaussian transmission showed that for nonzero flux in general there were more electrons scattered in one direction than the other. The expected value of the displacement and kinetic momentum along the ob- servation screen were likewise nonzero. Some workers $[4,5]$ have claimed that this calculation violates Ehrenfest's theorem and the conservation of momentum, because the assumption of Gaussian slits is unrealistic. In principle, however, there is nothing to prevent such slits from being prepared, although it would be difficult in practice. According to Olariu and Popescu [5], if a "realistic" calculation were made using rectangular slits, the AB diffraction pattern would be asymmetric, but with the expected displacement and kinetic momentum for the electron equal to zero in order to satisfy Ehrenfest's theorem $[4,5]$.

In this paper we show that for rectangular slits the $A B$ diffraction pattern is indeed asymmetric, with more electrons scattered in one direction than the other. Moreover, the expectation values of the displacement and momentum along the observation screen are nonzero. These results are not in conflict with Ehrenfest's theorem or momentum conservation, however. When an electron passes through either slit there is a force which acts on it at the slit edges. When magnetic flux is present, the problem no longer has reflectional symmetry, since the magnetic flux perpendicular to the plane can be either positive or negative. The geometry of the experiment is asymmetric, and therefore the diffraction pattern is also. More electrons can, indeed, be scattered in one direction than the other. The conservation of momentum is not violated because the screen with the slits is extremely massive and recoils without essentially changing its position. Each electron which passes through the slits is scattered either to the right or to the left. For each event, the screen with the slits recoils either to the left or to the right, respectively, to conserve momentum. Without magnetic flux the expected momentum, and therefore displacement, of the electrons along the screen is zero. With magnetic flux the expected momentum, and therefore the displacement, is nonzero. Therefore, the expected recoil momentum of the screen with the slits is equal and opposite.

In Sec. II the diffraction pattern is derived for rectangular slits from the Feynman path-integral technique. The asymmetry of the diffraction pattern and the expectation value of the displacement are calculated in Sec. III. 
In Sec. IV the problem is discussed from the standpoint of Ehrenfest's theorem. The conclusion is given in Sec. V.

\section{DIFFRACTION PATTERNS}

In this section we derive the single-slit wave function from the Feynman path-integral [6-8] approach to quantum mechanics. The principle of superposition is used to obtain the two-slit wave function. The diffraction pattern is proportional to the absolute value squared of the wave function.

\section{A. Single-slit diffraction pattern}

In the model considered here [6] the motion in the $y$ direction perpendicular to the screens is classical, while the motion in the $x$ direction parallel to the screens is quantum mechanical. When the Feynman propagators are used, the wave function at the observation screen can be calculated from the given initial wave function. For the single-slit geometry shown in Fig. 1, the wave function at the observation screen is

$$
\begin{aligned}
\Psi_{+}\left(x_{c}\right)=\int_{-\infty}^{+\infty} d x_{b} \int_{-\infty}^{+\infty} & d x_{a} K^{0}\left(x_{c}, x_{b}\right) G_{+}\left(x_{b}\right) \\
& \times K^{0}\left(x_{b}, x_{a}\right) \Psi_{a}\left(x_{a}\right) .
\end{aligned}
$$

The transmission function for the single slit of width $2 b$ whose center is at $x_{0}$ is

$$
G_{+}\left(x_{b}\right)= \begin{cases}0 & \text { if }\left|x_{b}-x_{0}\right|>b \\ 1 & \text { if }\left|x_{b}-x_{0}\right|<b\end{cases}
$$

The initial wave packet at $y=y_{a}$ and at time $t_{a}$ is taken to be a normalized Gaussian,

$$
\Psi_{a}\left(x_{a}\right)=\left(\frac{2 \alpha}{\pi}\right)^{1 / 4} \exp \left(-\alpha x_{a}^{2}\right)
$$

where $\alpha$ is a parameter which determines the width of the Gaussian. Instead of $\Psi_{a}$, Shapiro and Henneberger [8] chose a $\delta$ function, which is not normalizable. The free propagator $K^{0}\left(x_{c}, x_{b}\right)$ is defined as

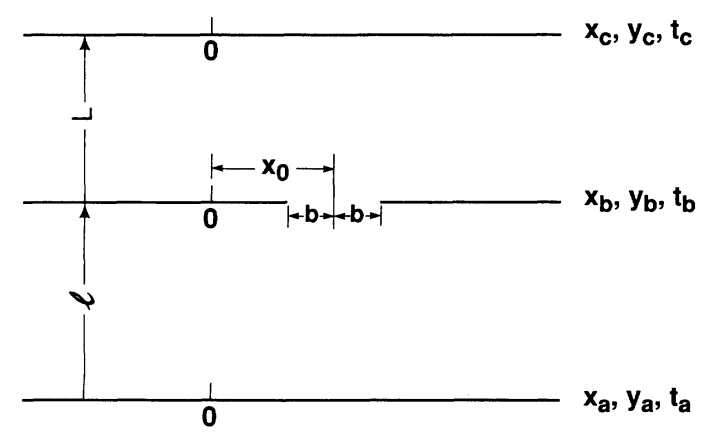

FIG. 1. The geometry of the single-slit diffraction experiment. The slit at $y_{b}$ has a width $2 b$ and is centered at $x_{b}=x_{0}$. The distances $L=y_{c}-y_{b}=v\left(t_{c}-t_{b}\right)$ and $l=y_{b}-y_{a}=v\left(t_{b}-t_{a}\right)$, where $v$ is the velocity in the $y$ direction, perpendicular to the screens, and $t_{a}, t_{b}, t_{c}$ are the times corresponding to the screens at $y_{a}, y_{b}, y_{c}$.
$K^{0}\left(x_{c}, x_{b}\right)=\left(\frac{m}{2 \pi i \hbar\left(t_{c}-t_{b}\right)}\right)^{1 / 2} \exp \left(\frac{i m}{2 \hbar} \frac{\left(x_{c}-x_{b}\right)^{2}}{\left(t_{c}-t_{b}\right)}\right)$,

where $m$ is the mass of the particle, $\hbar$ is Planck's constant $h$ divided by $2 \pi$, and the time variables in $K^{0}\left(x_{c}, x_{b}\right)$ are suppressed. A similar formula gives $K^{0}\left(x_{b}, x_{a}\right)$. In Eq. (1) the particle freely propagates from $y_{a}, x_{a}$ at time $t_{a}$ to the screen $y_{b}, x_{b}$ at time $t_{b}$, goes through the slit,. and propagates freely to the observation screen $y_{c}, x_{c}$ at time $t_{c}$.

When Eqs. (2) - (4) are substituted into Eq. (1), the integrals can be performed and the result is

$$
\begin{aligned}
\Psi_{+}\left(x_{c}\right)=- & i\left(\frac{2 \alpha}{\pi}\right)^{1 / 4}[2(F-i)]^{-1 / 2} \\
& \times \exp \left[i \alpha x_{c}^{2}(F+i)\left(F^{2}+1\right)^{-1}\right] \\
& \times\left[\operatorname{Ei}\left(\eta_{+}^{+}\right)-\operatorname{Ei}\left(\eta_{-}^{+}\right)\right] .
\end{aligned}
$$

The function $F$ is defined as

$$
F=\frac{\alpha \lambda(L+l)}{\pi},
$$

where $l=y_{b}-y_{a}$ is the distance in the $y$ direction from the source to the screen with the slits, and $L=y_{c}-y_{b}$ is the distance in the $y$ direction from the screen with the slits to the observation screen, as shown in Fig. 1. The de Broglie wavelength of the electron is $\lambda=h / m v$, where $v$ is the velocity of the particle in the $y$ direction. The complex Fresnel integral $\operatorname{Ei}(z)$ is defined as

$$
\operatorname{Ei}(z)=\int_{0}^{z} d \eta \exp \left(i \frac{\pi}{2} \eta^{2}\right) \text {. }
$$

The arguments of the complex Fresnel integral in Eq. (5) are

$$
\eta_{ \pm}^{+}=\beta\left(+x_{0} \pm b+\xi_{0}\right)
$$

where

$$
\begin{aligned}
& \beta=\left(\frac{2}{\lambda}\right)^{1 / 2}\left[L^{-1}+l^{-1}-(l D)^{-1}+i\left(\frac{\alpha \lambda}{\pi D}\right)\right]^{1 / 2}, \\
& \xi_{0}=\left[\frac{-x_{c}}{L}\right]\left[L^{-1}+l^{-1}-(l D)^{-1}+i\left(\frac{\alpha \lambda}{\pi D}\right)\right]^{-1},
\end{aligned}
$$

and

$$
D=1+\left(\frac{\lambda \alpha l}{\pi}\right)^{2}
$$

A plot of the diffraction pattern from a single slit of width $2 b$ with its center at $x_{b}=x_{0}$, which is proportional to $\left|\Psi_{+}\left(x_{c}\right)\right|^{2}$, is given in Fig. 2 .

\section{B. Two-slit diffraction pattern}

In the general treatment of the two-slit geometry shown in Fig. 3 all the paths leading from the origin to a point on the screen at $x_{c}, y_{c}$ should be taken into account. 


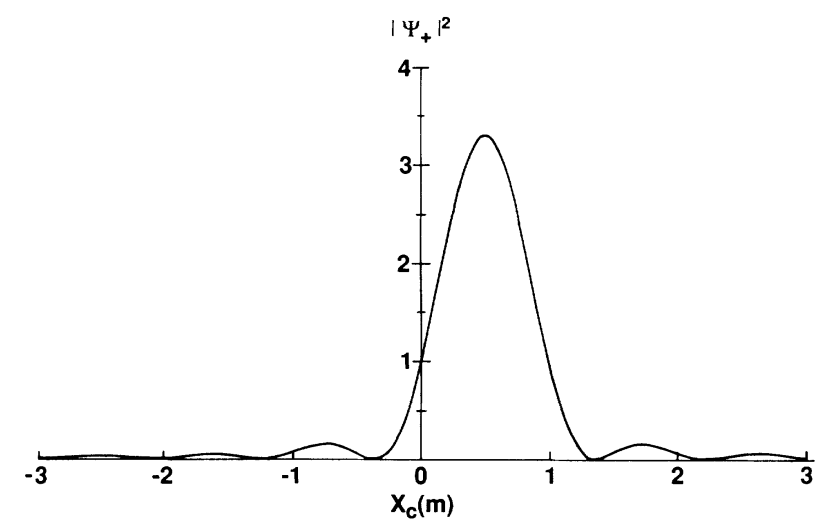

FIG. 2. A plot of the single-slit diffraction pattern, which is proportional to $\left|\Psi_{+}\left(x_{c}\right)\right|^{2}$ [see Eq. (5)]. The parameters chosen are $l=10 \mathrm{~m}, L=1 \mathrm{~m}, \alpha=10 \mathrm{~m}^{-2}, x_{0}=0.5 \mathrm{~m}, b=1.0 \times 10^{-9} \mathrm{~m}$, and $\lambda=1.74 \times 10^{-9} \mathrm{~m}$.

However, if the width of the slits is small compared to their distance of separation, the paths which have higher winding numbers around the magnetic flux should be negligible. For example, a path that passes through one slit, loops around the magnetic flux, goes through the other slit in the opposite direction, and finally goes again through the original slit, would not be likely. In our model [6], we only consider paths which go once through a slit. We replace the two-dimensional path integral with a one-dimensional path integral, where the particle moves quantum mechanically in the $x$ direction and classically in the $y$ direction. In this model the possibility of higher winding numbers is neglected.

The two-slit diffraction pattern for the geometry in Fig. 3 is obtained from Eq. (1) with the single-slit transmission function $G_{+}\left(x_{b}\right)$ replaced by the two-slit transmission function $G_{+}\left(x_{b}\right)+G_{-}\left(x_{b}\right)$, where $G_{ \pm}\left(x_{b}\right)$ is the transmission function for the slit at $\pm x_{0}$. In the absence of magnetic flux, the corresponding wave function $\Psi\left(x_{c}\right)$ (unnormalized) at the observation screen is

$$
\Psi\left(x_{c}\right)=\Psi_{+}\left(x_{c}\right)+\Psi_{-}\left(x_{c}\right),
$$

where $\Psi_{ \pm}\left(x_{c}\right)$ is the contribution to the wave function corresponding to the slit at $\pm x_{0}$ given in Eq. (1). The

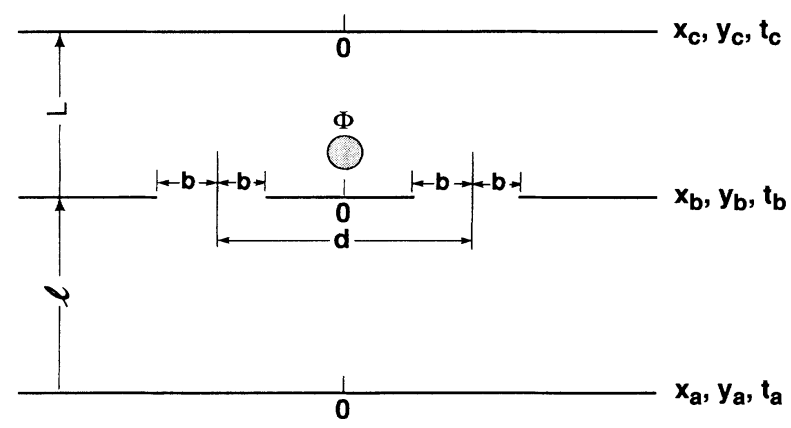

FIG. 3. The geometry of the two-slit diffraction experiment. Magnetic flux $\Phi$ is present in a shielded solenoid at $x_{b}=0$. The slits of width $2 b$ are centered at $\pm x_{0}$, and are separated by a distance $d=2 x_{0}$. The distances $L$ and $l$ are defined in Fig. 1 . contribution $\Psi_{-}\left(x_{c}\right)$ is given by $\Psi_{+}\left(x_{c}\right)$ in Eq. (1) with $+x_{0}$ changed to $-x_{0}$. From Eq. (1) we see that $\Psi_{-}\left(x_{c}\right)=\Psi_{+}\left(-x_{c}\right)$, since $G_{-}\left(x_{b}\right)=G_{+}\left(-x_{b}\right)$. An interference pattern is observed on the screen at $y_{c}$ because the two waves superimposed in Eq. (12) are coherent.

If magnetic flux $\Phi$ is present in an inaccessible region between the two slits, then a vector potential exists in the region accessible to the electron. A detailed analysis of the Feynman path integral [6] shows that the wave function at the observation screen when only the slit at $\pm x_{0}$ is open is

$$
\Psi_{ \pm}^{\prime}\left(x_{c}\right)=\exp \left(i \frac{q}{\hbar c} \int_{C_{ \pm}} \mathbf{A} \cdot d \mathbf{r}\right) \Psi_{ \pm}\left(x_{c}\right)
$$

where $q<0$ is the charge of the electron, $\mathbf{A}$ is the vector potential, and $C_{+}\left(C_{-}\right)$is the path which goes from $y_{a}, x_{a}$ through the slit at $+x_{0}\left(-x_{0}\right)$ to $y_{c}, x_{c}$.

When both slits are open the transmission function is $G_{+}\left(x_{b}\right)+G_{-}\left(x_{b}\right)$ in the Feynman path integral, which gives the total wave function $\Psi^{\prime}\left(x_{c}\right)$ on the observation screen as a coherent superposition

$$
\begin{aligned}
\Psi^{\prime}\left(x_{c}\right)= & \Psi_{+}^{\prime}\left(x_{c}\right)+\Psi_{-}^{\prime}\left(x_{c}\right) \\
= & \exp \left[i \frac{q}{\hbar c} \int_{C_{+}} \mathbf{A} \cdot d \mathbf{r}\right] \\
& \times\left[\Psi_{+}\left(x_{c}\right)+\exp (-i \phi) \Psi_{-}\left(x_{c}\right)\right],
\end{aligned}
$$

where the flux parameter $\phi=q \Phi / \hbar c$. By Stokes's theorem the magnetic flux $\Phi$ enclosed between the two slits is

$$
\Phi \equiv \int_{S} \mathbf{B} \cdot d \mathbf{a}=\oint_{C} \mathbf{A} \cdot d \mathbf{r}
$$

where $d \mathbf{a}$ is an element of area of the surface $S$ which has $C$ as its boundary. The path $C=C_{+}-C_{-}$, which encloses the flux, goes from the origin at $x_{a}, y_{a}$ through the slit at $+x_{0}, y_{b}$ to the observation screen at $x_{c}, y_{c}$, and returns through the slit at $-x_{0}, y_{b}$ to the origin.

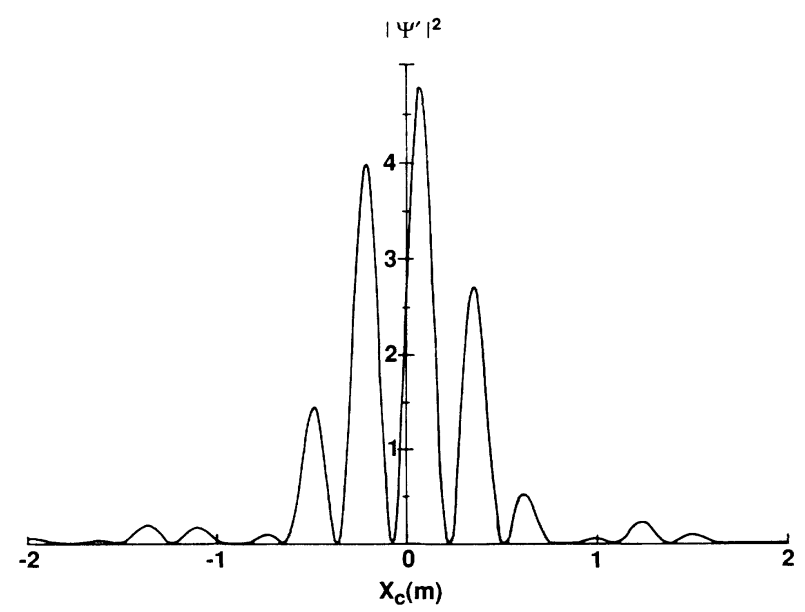

FIG. 4. A plot of the two-slit diffraction pattern, which is proportional to $\left|\Psi^{\prime}\left(x_{c}\right)\right|^{2}$ in Eq. (16). The parameters chosen are $l=10 \mathrm{~m}, L=1 \mathrm{~m}, \alpha=10 \mathrm{~m}^{-2}, x_{0}=3.0 \times 10^{-9} \mathrm{~m}$, $b=1.0 \times 10^{-9} \mathrm{~m}, \phi=\pi / 2$, and $\lambda=1.74 \times 10^{-9} \mathrm{~m}$. 
In the presence of magnetic flux the two-slit diffraction pattern on the observation screen at $y_{c}$ is

$$
\begin{aligned}
\left|\Psi^{\prime}\left(x_{c}\right)\right|^{2}= & \left|\Psi_{+}\left(x_{c}\right)\right|^{2}+\left|\Psi_{-}\left(x_{c}\right)\right|^{2} \\
& +2 \cos \phi \operatorname{Re} \Psi_{+}^{*}\left(x_{c}\right) \Psi_{-}\left(x_{c}\right) \\
& +2 \sin \phi \operatorname{Im} \Psi_{+}^{*}\left(x_{c}\right) \Psi_{-}\left(x_{c}\right) .
\end{aligned}
$$

A plot of the diffraction pattern in Eq. (16) is given in Fig. 4.

\section{EXPECTATION VALUES}

The wave function obtained in Sec. II for the two-slit geometry with nonzero magnetic flux between the slits is used here to calculate expectation values of observables. The first quantity calculated is the asymmetry factor, which is a measure of the relative number of particles scattered to the right-hand side as compared to the left. The second quantity calculated is the average displacement along the observation screen.

\section{A. Asymmetry parameter}

The asymmetry parameter $r$ is defined as

$$
r=\frac{A_{R}-A_{L}}{A_{R}+A_{L}}
$$

where $A_{R}\left(A_{L}\right)$ is the area of the diffraction pattern to the right (left) of the origin. Equation (17) can be considered as the expectation value of $\epsilon\left(x_{c}\right)=1$ for $x_{c}>0$ and -1 for $x_{c}<0$, with respect to the wave function $\Psi^{\prime}$ in Eq. (14).

With magnetic flux between the slits the area to the right of the origin for the two-slit diffraction pattern is

$$
\begin{aligned}
A_{R}=\int_{0}^{\infty} d x_{c} \mid & \left.\Psi^{\prime}\left(x_{c}\right)\right|^{2} \\
=\int_{0}^{\infty} d x_{c}[ & \left|\Psi_{+}\left(x_{c}\right)\right|^{2}+\left|\Psi_{-}\left(x_{c}\right)\right|^{2} \\
& +2 \cos \phi \operatorname{Re} \Psi_{+}^{*}\left(x_{c}\right) \Psi_{-}\left(x_{c}\right) \\
& \left.+2 \sin \phi \operatorname{Im} \Psi_{+}^{*}\left(x_{c}\right) \Psi_{-}\left(x_{c}\right)\right] .
\end{aligned}
$$

The area to the left is

$$
\begin{aligned}
A_{L}=\int_{0}^{\infty} d x_{c}[ & \left|\Psi_{+}\left(x_{c}\right)\right|^{2}+\left|\Psi_{-}\left(x_{c}\right)\right|^{2} \\
& +2 \cos \phi \operatorname{Re} \Psi_{+}^{*}\left(x_{c}\right) \Psi_{-}\left(x_{c}\right) \\
& \left.-2 \sin \phi \operatorname{Im} \Psi_{+}^{*}\left(x_{c}\right) \Psi_{-}\left(x_{c}\right)\right],
\end{aligned}
$$

where we have used $\Psi_{-}\left(x_{c}\right)=\Psi_{+}\left(-x_{c}\right)$.

The asymmetry factor in Eq. (17) can therefore be rewritten as

$$
r=\frac{a \sin \phi}{1+\gamma \cos \phi}
$$

where the constants $a$ and $\gamma$ are defined as

$$
a=2 \int_{0}^{\infty} d x_{c} \operatorname{Im} \Psi_{+}^{*}\left(x_{c}\right) \Psi_{-}\left(x_{c}\right) /\left\langle\Psi_{+} \mid \Psi_{+}\right\rangle
$$

and

$$
\gamma=2 \int_{0}^{\infty} d x_{c} \operatorname{Re} \Psi_{+}^{*}\left(x_{c}\right) \Psi_{-}\left(x_{c}\right) /\left\langle\Psi_{+} \mid \Psi_{+}\right\rangle .
$$

For $l=10 \mathrm{~m}, L=1 \mathrm{~m}, \alpha=10 \mathrm{~m}^{-2}, x_{0}=3 \times 10^{-9} \mathrm{~m}$, $b=1.0 \times 10^{-9} \mathrm{~m}$, and $\lambda=1.74 \times 10^{-9} \mathrm{~m}$ the value of $a=0.2622$ and $\gamma=3.76 \times 10^{-5}$. For $\phi=\pi / 2$ the value of $r=0.2622$. From Eq. (20) we see that $r$ is positive for $0<\phi<\pi$ and more particles are diffracted to the right than to the left.

\section{B. Expected displacement}

Even though the diffraction pattern in Fig. 4 shows an asymmetry, several workers [3-5] claim that the expected displacement in the $x_{c}$ direction is still zero for all $\phi$. In this subsection we show that the expected displacement is in general nonzero.

The expected displacement in the $x_{c}$ direction is

$$
\left\langle x_{c}\right\rangle=\left\langle\Psi^{\prime}\left|x_{c}\right| \Psi^{\prime}\right\rangle /\left\langle\Psi^{\prime} \mid \Psi^{\prime}\right\rangle
$$

for the wave function $\Psi^{\prime}$ in Eq. (14). When Eq. (14) is substituted into Eq. (23) and the result is simplified, we obtain

$$
\left\langle x_{c}\right\rangle=\frac{c \sin \phi}{1+\gamma \cos \phi},
$$

where

$$
c=2 \int_{0}^{\infty} d x_{c} x_{c} \operatorname{Im} \Psi_{+}^{*}\left(x_{c}\right) \Psi_{-}\left(x_{c}\right) /\left\langle\Psi_{+} \mid \Psi_{+}\right\rangle
$$

and $\gamma$ is given in Eq. (22).

For $l=10 \mathrm{~m}, L=1 \mathrm{~m}, \alpha=10 \mathrm{~m}^{-2}, x_{0}=3.0 \times 10^{-9} \mathrm{~m}$, $b=1.0 \times 10^{-9} \mathrm{~m}$, and $\lambda=1.74 \times 10^{-9} \mathrm{~m}$, the value of $c=6.424 \times 10^{-4} \mathrm{~m}$. For $\phi=\pi / 2$ the value of $\left\langle x_{c}\right\rangle$ $=6.424 \times 10^{-4} \mathrm{~m}$. From Eq. (24) we see that $\left\langle x_{c}\right\rangle$ is positive for $0<\phi<\pi$.

\section{EHRENFEST'S THEOREMS}

In this section Ehrenfest's theorems and their relationship to the Aharonov-Bohm effect are discussed.

\section{A. Kinematical equation}

The time rate of change of Eq. (23) gives the kinematical Ehrenfest theorem,

$$
\frac{d\left\langle x_{c}\right\rangle}{d t_{c}}=\frac{\left\langle\pi_{c}\right\rangle}{m} .
$$

The kinetic momentum operator $\pi_{c}$ is defined as

$$
\pi_{c}=p_{c}-q A_{x}\left(x_{c}, t_{c}\right),
$$

where the canonical momentum operator is $p_{c}=-i \hbar \partial / \partial x_{c}$. The expectation value of $\pi_{c}$ in Eq. (26) with respect to the wave function $\Psi^{\prime}$ in Eq. (14) is

$$
\left\langle\pi_{c}\right\rangle=\left\langle\Psi^{\prime}\left|\pi_{c}\right| \Psi^{\prime}\right\rangle /\left\langle\Psi^{\prime} \mid \Psi^{\prime}\right\rangle .
$$

The right-hand side of Eq. (26) is the expected velocity in the $x_{c}$ direction.

The normalization integral $\left\langle\Psi^{\prime} \mid \Psi^{\prime}\right\rangle$ is a constant in time because $\Psi^{\prime}=\Psi^{\prime}\left(x_{c}, t_{c}\right)$ satisfies the time-dependent Schrödinger equation

$$
H\left(x_{c}, t_{c}\right) \Psi^{\prime}\left(x_{c}, t_{c}\right)=i \hbar \frac{\partial \Psi^{\prime}\left(x_{c}, t_{c}\right)}{\partial t_{c}} .
$$


The Hamiltonian is

$$
H\left(x_{c}, t_{c}\right)=\pi_{c}^{2} / 2 m,
$$

because there is no potential energy at the observation screen at $x_{c}, y_{c}$ at time $t_{c}$.

\section{B. Dynamical equation}

The dynamical Ehrenfest theorem is obtained by taking the time derivative of Eq. (28),

$$
\frac{d\left\langle\pi_{c}\right\rangle}{d t_{c}}=0,
$$

which shows that the expected force on the electron is zero, in agreement with Eq. (30).

Integration of Eq. (31) shows that $\left\langle\pi_{c}\right\rangle$ is a constant, and consequently the expected velocity in Eq. (26) is a constant. If we integrate Eq. (26) from $t_{b}$ to $t_{c}$, we obtain

$$
\left\langle x_{c}\right\rangle=\left\langle\pi_{c}\right\rangle\left(t_{c}-t_{b}\right) / m
$$

since no force acts on the electron during its propagation from $y_{b}, x_{b}$ at time $t_{b}$ to its final position at $y_{c}, x_{c}$ at time $t_{c}$. Therefore a nonzero expected displacement $\left\langle x_{c}\right\rangle \mathrm{im}$ plies a nonzero expected kinetic momentum $\left\langle\pi_{c}\right\rangle$ in the $x_{c}$ direction. For Eq. (32) to be valid it is necessary that $\left.\left.\left|\left\langle x_{c}\right\rangle\right|\right\rangle\right\rangle d=2 x_{0}$, the distance between the slits, and that $L \gg d$.

\section{Discussion}

Since no Lorentz force acts on the electron, it seems intuitively obvious that the expected kinetic momentum $\left\langle\pi_{c}\right\rangle$ and displacement $\left\langle x_{c}\right\rangle$ are both zero for all values of the magnetic flux, as claimed in [3-5]. Bocchieri and Loinger [9] have gone so far as to say that the asymmetry of the $A B$ diffraction pattern is an artifact of the mathematics, because it violates their physical intuition.

If we consider diffraction in the absence of magnetic flux, the symmetry of the situation implies that $\left\langle x_{c}\right\rangle$ and $\left\langle\pi_{c}\right\rangle$ are both zero. Nevertheless, each electron which passes through the slits will be scattered to the right or to the left. The force which causes this scattering is due to the edges of the slits, since the slits can be described by an infinitely high potential everywhere, except at $\left|x_{b} \pm x_{0}\right|<b$, where the potential is zero. In each scattering event the linear momentum must be conserved. If an electron is scattered to the right, for example, the screen with the slits must recoil to the left to conserve momentum. Since the screen with the slits is extremely massive, it recoils without essentially changing its position. The electron-diffraction pattern is symmetric, so as many electrons are scattered to the right as to the left, and the expected displacement $\left\langle x_{c}\right\rangle$ and momentum $\left\langle\pi_{c}\right\rangle$ of the electron are zero. Therefore, the expected recoil momentum $-\left\langle\pi_{c}\right\rangle$ is also zero.

In the presence of magnetic flux in an inaccessible region between the two slits, the symmetry of the situation is destroyed, since the magnetic flux can be positive or negative. The diffraction pattern in general becomes asymmetric due to the Aharonov-Bohm effect, so more electrons are scattered to the right, say, than to the left. As this paper shows, the expectation values of $\left\langle x_{c}\right\rangle$ and $\left\langle\pi_{c}\right\rangle$ are nonzero. For each electron scattered to the right or to the left by the edges of the slits, linear momentum is conserved by the recoil of the screen with the slits to the left or to the right, respectively. Since the experimental arrangement is no longer symmetrical because of the magnetic flux, the nonzero average kinetic momentum $\left\langle\pi_{c}\right\rangle$ of the electron implies that the screen with the slits recoils by $-\left\langle\pi_{c}\right\rangle$ to conserve momentum. Thus the nonzero values of $\left\langle x_{c}\right\rangle$ and $\left\langle\pi_{c}\right\rangle$ are completely compatible with momentum conservation and no Lorentz force. The Aharonov-Bohm effect is a global (or nonlocal) effect, in the sense that the diffraction pattern is asymmetric even though the magnetic flux is in an inaccessible region.

\section{Critique of previous work}

Since the conservation laws are explicitly given in terms of the density of observables like displacement, velocity, energy, electric field, and magnetic field, Olariu and Popescu [5] conclude that there can be no effect of the flux on these quantities. This conclusion overlooks the fact that the wave function $\Psi^{\prime}$ in Eq. (14) depends on the enclosed magnetic flux, so therefore the density of an observable $A$, which is $\operatorname{Re} \Psi^{\prime *} A \Psi^{\prime} /\left\langle\Psi^{\prime} \mid \Psi^{\prime}\right\rangle$, can also depend on the enclosed flux. The wave function $\Psi^{\prime}$ satisfies the Schrödinger equation, in which the Hamiltonian depends on the flux through the dependence of the kinetic momentum.

An oversimplified argument is given by Olariu and Popescu [5] for why the expected displacement $\left\langle x_{c}\right\rangle$ must be zero. Their argument is similar to one due to Aharonov, et al. [4]. In our notation their argument is the following. If $\widetilde{\Psi}_{ \pm}\left(x_{b}, t_{b}\right)$ is the wave function defined between $\pm x_{0}-b$ and $\pm x_{0}+b$, then it is clear that $\widetilde{\Psi}_{+}^{*}\left(x_{b}, t_{b}\right) \widetilde{\Psi}_{-}\left(x_{b}, t_{b}\right)=0$, so that the integrals

$$
\int_{-\infty}^{+\infty} d x_{b} \widetilde{\Psi}_{+}^{*}\left(x_{b}, t_{b}\right) x_{b} \widetilde{\Psi}_{-}\left(x_{b}, t_{b}\right)=0
$$

and

$$
\int_{-\infty}^{+\infty} d x_{b} \widetilde{\Psi}_{+}^{*}\left(x_{b}, t_{b}\right) \pi_{b} \widetilde{\Psi}_{-}\left(x_{b}, t_{b}\right)=0 .
$$

In order to obtain $\widetilde{\Psi}_{ \pm}\left(x_{c}, t_{c}\right)$, the corresponding wave function on the observation screen, it is necessary to use Eq. (1), in which the electron is propagated forward both in space and time. Olariu and Popescu [5] only consider $\widetilde{\Psi}_{ \pm}\left(x_{b}, t_{c}\right)$, the wave function at the slits at the later time $t_{c}$. In that case Eqs. (33) and (34) are still satisfied for $t_{c}$. They use the principle of superposition to give the total wave function on the screen with the slits, which is

$$
\widetilde{\Psi}\left(x_{b}, t_{c}\right)=\widetilde{\Psi}_{+}\left(x_{b}, t_{c}\right) e^{i \phi_{+}}+\widetilde{\Psi}_{-}\left(x_{b}, t_{c}\right) e^{i \phi_{-}},
$$

where $\phi_{+}$and $\phi_{-}$are the phases due to the vector potential. Then they show that $\left\langle\widetilde{\Psi}\left|x_{b}\right| \widetilde{\Psi}\right\rangle$ at time $t_{c}$ does not depend on the flux, because the interference term vanishes.

The correct procedure requires the use of the Feynman path integral in Eq. (1) in which the wave function is propagated forward in both space and time. According to 
Eqs. (24) and (32) we then have nonzero values of $\left\langle x_{c}\right\rangle$ and $\left\langle\pi_{c}\right\rangle$ at the observation screen.

\section{CONCLUSION}

The presence of magnetic flux in an inaccessible region between the two slits gives in general an asymmetric electron-diffraction pattern, which has a nonzero expectation value for the displacement $\left\langle x_{c}\right\rangle$ and kinetic momentum $\left\langle\pi_{c}\right\rangle$. Others [3-5] have agreed that the diffraction pattern is asymmetric, but have argued that for the diffraction pattern, $\left\langle\pi_{c}\right\rangle$, and consequently $\left\langle x_{c}\right\rangle$, must be zero from the conservation of momentum.

The nonzero values of $\left\langle x_{c}\right\rangle$ and $\left\langle\pi_{c}\right\rangle$ do not violate any conservation laws. The geometry of the experiment is asymmetric because the magnetic flux can be either positive or negative. There is no Lorentz force, but a force acts on the electron when it goes through the two slits in the screen at $y_{b}$. At the edges of the slits there is an infinite discontinuity in the potential, and hence a force acts on the electron. The screen recoils every time an electron goes through it in order to conserve momentum for each electron. Because the screen is extremely massive, it recoils without essentially changing its position. The total energy of the electron is also conserved. The increase in the energy corresponding to the motion in the $x_{c}$ direction is just compensated by a decrease in the energy corresponding to the motion in the $y$ direction.

In this paper it is shown that a nonzero expected displacement $\left\langle x_{c}\right\rangle$ and kinetic momentum $\left\langle\pi_{c}\right\rangle$ are obtained with rectangular slits, just as they were for Gaussian slits [6]. The nonzero values are not due to any approximations within the model considered and are consistent with the conservation laws, which also hold in quantum theory.

The nonzero values of $\left\langle x_{c}\right\rangle$ and $\left\langle\pi_{c}\right\rangle$ obtained here are counterintuitive, since some workers who accept the asymmetric $\mathrm{AB}$ diffraction pattern have maintained (without adequate substantiation) that they are zero [3-5]. The AB effect, and hence quantum mechanics, is even stranger than many have realized.

\section{ACKNOWLEDGMENTS}

We would like to thank Josefina Pérez Alvaréz for advice on the numerical and computational aspects of this work. D.H.K. is supported in part by the Fulbright Commission in Brazil and a University of North Texas Faculty Development Grant. V.C.A.-N. is supported in part by CNPq, Brazil and R.M.R. is supported by CNPq, Brazil.
[1] Y. Aharonov and D. Bohm, Phys. Rev. 115, 485 (1959).

[2] T. T. Wu and C. N. Yang, Phys. Rev. D 12, 3845 (1975).

[3] T. H. Boyer, Phys. Rev. D 8, 1679 (1973).

[4] Y. Aharonov, C. K. Au, E. C. Lerner, and J. Q. Liang, Lett. Nuovo Cimento 39, 145 (1984).

[5] S. Olariu and I. I. Popescu, Rev. Mod. Phys. 57, 339 (1979).
[6] D. H. Kobe, Ann. Phys. (N.Y.) 123, 381 (1979).

[7] R. P. Feynman and A. R. Hibbs, Quantum Mechanics and Path Integrals (McGraw Hill, New York, 1965).

[8] D. Shapiro and W. C. Henneberger, J. Phys. A 22, 3605 (1989).

[9] P. Bocchieri and A. Loinger, Lett. Nuovo Cimento 35, 469 (1982). 


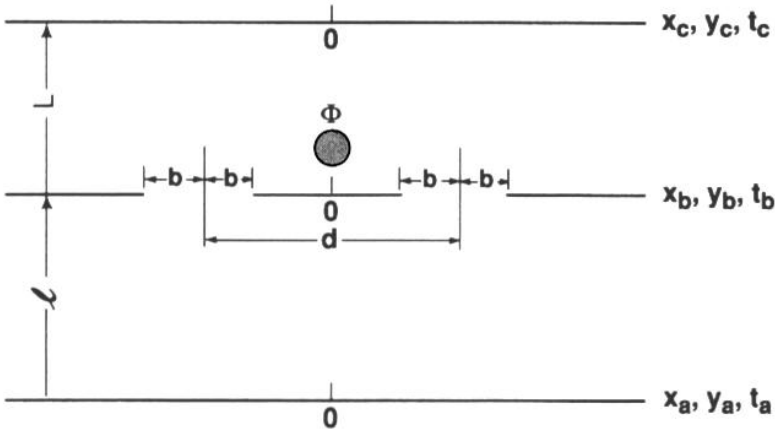

FIG. 3. The geometry of the two-slit diffraction experiment. Magnetic flux $\Phi$ is present in a shielded solenoid at $x_{b}=0$. The slits of width $2 b$ are centered at $\pm x_{0}$, and are separated by a distance $d=2 x_{0}$. The distances $L$ and $l$ are defined in Fig. 1 . 